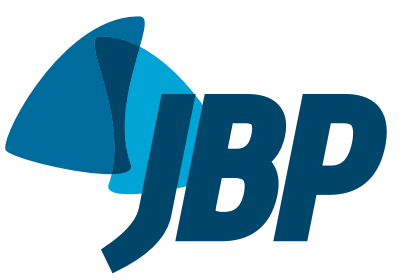

A 49-year-old male smoker (with a smoking history of 90 pack-years) presented with vague complaints of dyspnea. Laboratory test results were normal. Serology for HIV was negative. A CT scan of the chest showed a nodule with the halo sign in the left lower lobe (Figure 1). Fungal serology was negative.

The halo sign is a nonspecific CT finding consisting of a halo of ground-glass opacity surrounding a nodule or, less commonly, a mass or a rounded area of consolidation.

In most cases, a halo of ground-glass opacity represents perinodular hemorrhage. In cases of angioinvasive aspergillosis (AIA), for example, a nodule represents pulmonary infarction secondary to fungal angioinvasion and a surrounding halo of ground-glass opacity represents perinodular alveolar hemorrhage. In other infectious processes, the halo sign represents perilesional inflammatory infiltration. In cases of adenocarcinoma, the halo sign represents tumor cell proliferation along the alveolar septa, the pulmonary architecture being preserved (lepidic growth). The same features can be seen in some cases of metastatic adenocarcinoma (particularly in cases of adenocarcinoma originating from the gastrointestinal tract or pancreas).

As an initial diagnostic approach, it is useful to determine patient immune status. In immunocompromised patients, the halo sign is most commonly due to infectious diseases, the most common being invasive fungal diseases, such as AIA. Therefore, in the presence of febrile neutropenia,

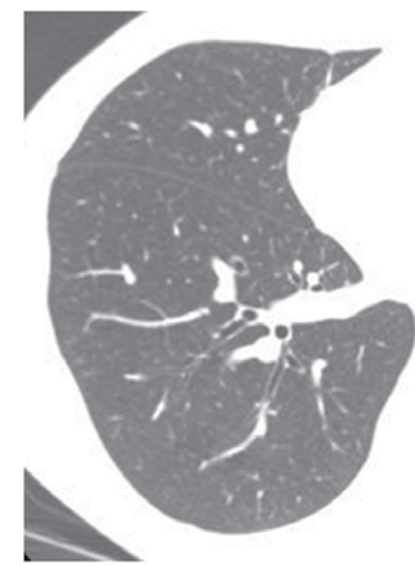

Figure 1. CT scan of the chest with lung window settings at the level of the lower lobes, showing a soft-tissue density nodule located in the left lower lobe and surrounded by a halo of ground-glass opacity (the halo sign).

\section{RECOMMENDED READING}

. Fraser RS, Müller NL, Colman NC, Pare PD, editors. Diagnosis of Diseases of the Chest. 4th ed. Philadelphia: WB Saunders Company; 1999.

1. Universidade Federal Fluminense, Niterói (RJ) Brasil.

2. Universidade Federal do Rio de Janeiro, Rio de Janeiro (RJ) Brasil.

3. Santa Casa de Misericórdia de Porto Alegre, Porto Alegre (RS) Brasil.

4. Universidade Federal de Ciências da Saúde de Porto Alegre, Porto Alegre (RS) Brasil.

5. Faculdade de Medicina de Petrópolis, Petrópolis (RJ) Brasil. especially in patients with hematological malignancies and in bone marrow transplant recipients, AIA is the major cause of the halo sign. In such cases, the halo sign is considered to constitute early evidence of AIA, warranting initiation of antifungal therapy before serologic test results are known. Early diagnosis is essential because the disease is associated with high mortality rates. In immunocompetent patients, especially asymptomatic patients and smokers, bronchial carcinoma, particularly lepidic adenocarcinoma (formerly known as bronchioloalveolar carcinoma), is the major cause of the halo sign. The differential diagnosis should also include the following: hemorrhagic metastases, particularly those related to angiosarcomas and choriocarcinomas; lymphomas; Kaposi's sarcoma; bacterial infections (including tuberculosis and actinomycosis); fungal infections (including candidiasis, mucormycosis, cryptococcosis, histoplasmosis, and paracoccidioidomycosis); viral infections (including infection with cytomegalovirus, herpes simplex virus, and varicella-zoster virus); sarcoidosis; granulomatosis with polyangiitis (Wegener's granulomatosis); and organizing pneumonia.

Although the halo sign is a nonspecific finding with a broad differential diagnosis, the differential diagnosis can be considerably narrowed by correlating clinical data, laboratory test results, and associated CT findings. However, in many cases, a final diagnosis requires histopathological examination. In our patient, a biopsy revealed adenocarcinoma with a lepidic growth pattern.

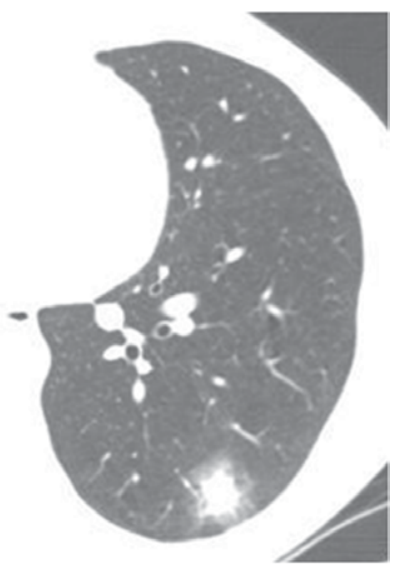




\section{ERRATUM}

Manuscript: The halo sign.

Publication: J Bras Pneumol. 2017;43(1):4-4.

On the original publication "The halo sign", in its English version, 2017, n.43, v.1, p.4, where it is written DOI: http://dx.doi.org/10.1590/s1806-37562016000000354

It should read DOI:

http://dx.doi.org/10.1590/s1806-37562016000000324 